\title{
Asymptomatic Plasmodium vivax parasitaemia in the low-transmission setting: the role for a population-based transmission-blocking vaccine for malaria elimination
}

\author{
Thomas C. S. Martin and Joseph M. Vinetz ${ }^{*}$ (])
}

\begin{abstract}
Plasmodium vivax remains an important cause of morbidity and mortality across the Americas, Horn of Africa, East and South East Asia. Control of transmission has been hampered by emergence of chloroquine resistance and several intrinsic characteristics of infection including asymptomatic carriage, challenges with diagnosis, difficulty eradicating the carrier state and early gametocyte appearance. Complex human-parasite-vector immunological interactions may facilitate onward infection of mosquitoes. Given these challenges, new therapies are being explored including the development of transmission to mosquito blocking vaccines. Herein, the case supporting the need for transmissionblocking vaccines to augment control of P. vivax parasite transmission and explore factors that are limiting eradication efforts is discussed.
\end{abstract}

Keywords: Plasmodium vivax, Transmission blocking vaccine, Premonition, Asymptomatic parasitaemia

\section{Background}

At a time when malaria eradication is advocated as the ultimate goal of malaria control strategies worldwide, the mechanisms by which malaria remains endemic in the low-transmission setting remain unclear, and hence novel approaches to regional malaria elimination are difficult to approach. The 2017 World Malaria Report notes that in the Americas, 12 of the 18 malaria-endemic countries are projected to reduce malaria case incidence by at least $40 \%$ by 2020 , but that malaria cases increased in 4 (Nicaragua, Panama, Peru and Venezuela) in the period 2010-2016 [1]. Brazil and Venezuela accounted for about two-thirds of reported cases in the Americas, and the increase in Peruvian malaria cases after 2009 has reversed gains since 2000 [1]. These trends yet again

\footnotetext{
*Correspondence: jvinetz@ucsd.edu

Division of Infectious Diseases, Department of Medicine, University of California San Diego, La Jolla, CA, USA
}

demonstrate the fragility of malaria control and elimination efforts that are subject to the vagaries of political and socio-demographic trends.

In areas of relatively low levels of transmission, such as the Amazon basin, Plasmodium vivax causes considerable morbidity, similar to many other regions such as those in the Horn of Africa, south and southeast Asia [1]. The geographic range of $P$. vivax malaria is broader than that of Plasmodium falciparum malaria, yet both declined considerably during the 20th century, especially over the past decade. Given continued global population growth, the global population at risk for malaria now estimated at 3.5 billion people [1]. The WHO estimates that from this pool the current number of new $P$. vivax infections is in the range 6.6-10.8 million cases per year with roughly 3000 deaths; however, previous studies have suggested that this is a dramatic underestimate with annual global cases of vivax malaria possibly being as high as 391 million $[1,2]$. 
To date the most effective approaches to malaria control have been through direct and indirect interventions against the mosquito vector. Vector control in the 20th century though improvements in sanitation and the introduction of dichlorodiphenyltrichloroethane (DDT) in the 1940s (but stopped in the 1970s-1980s in South America [3-5]), combined with effective chemotherapy and chemoprevention dramatically reduced the reach of $P$. vivax malaria. However, several key host and parasite characteristics of $P$. vivax malaria have enabled this parasite to maintain endemicity, including the emergence of infectious gametocytes at the very onset of parasitaemia, the development of hypnozoites as latent liver infections in humans that later relapse, and asymptomatic parasitaemia [6-13]. Efforts to reduce the impact of endemic $P$. vivax have not established an effective way to manage ongoing transmission through relapsed infections and asymptomatic infections, especially as chloroquineresistant $P$. vivax has spread, particularly in the AsiaPacific region [10, 14]. Potential interventions to reduce transmission from both latently and subclinically lowlevel parasitaemic individuals include mass drug administration or vaccination. Given these considerations new conceptual approaches and tools to advance malaria control measures from control to elimination must still be advanced. Here the parasite and host characteristics that underlie the maintenance of regional $P$. vivax hypoendemicity, from which potential interventions, particularly transmission-blocking vaccine approaches, emerge as promising strategies are considered.

\section{Plasmodium vivax life cycle with regard to transmission-blocking vaccine development}

Several aspects of the life cycle of $P$. vivax allow it to maintain endemicity but one of the most formidable is the ability to develop latent infection as hypnozoites [15]. Upon initial infection of humans, sporozoites migrate to the liver where they infect hepatocytes becoming either tissue schizonts (exoerythrocytic forms, EEFs), leading to blood stage malaria, or hypnozoites, a dormant form of the parasite that can reactivate weeks, months or years later $[15,16]$. Parasite dormancy can lead to reemergence of active infections despite active populationbased vector control programmes. As the vast majority of $P$. vivax relapses are likely asymptomatic $[17,18]$, most do not come to medical attention and are consequently important potential sources to maintain endemicity and reintroduce $P$. vivax to human populations.

Once introduction of infection to a population occurs, diagnosis remains a challenge in some people. During the initial stages of infection, each EEF releases tens of thousands of merozoites into the blood stream, which preferentially infect reticulocytes over mature red cells.
Hence infected people typically have lower parasite densities than those infected by P. falciparum, which invades red cells of all ages. These low level parasitaemias with $P$. vivax can lead to infections being missed by routine light microscopy or rapid diagnostic tests. This in turn means some individuals do not receive timely treatment, thus permitting ongoing transmission.

In contrast to $P$. falciparum, $P$. vivax gametocytes are sensitive to blood schizonticides as evidenced by the rapid disappearance of all parasite stages after chloroquine treatment. But, infectious gametocytes of $P$. vivax appear early in the course of infection, simultaneously with asexual forms. As a result, transmission of infection to anopheline mosquitoes can occur before adequate gametocidal therapy can be initiated. Then, after ingestion of an infection blood meal, sporogony occurs within the mosquito in which development can occur at lower temperatures than for other Plasmodium species, explaining its broader geographical spread and longer transmission seasons.

\section{Current treatment of acute, symptomatic Plasmodium vivax malaria and radical cure}

Acute $P$. vivax malaria is conventionally treated with a blood schizonticide (i.e. chloroquine, mefloquine, atovaquone/proguanil) followed by radical cure with the 8-aminoquinoline, primaquine, initiated simultaneously. Challenges have arisen with treatment including clinically significant chloroquine-resistant $P$. vivax that has emerged in Asia, the Brazilian Amazon and Oceania [10, $19,20]$. In addition, despite treatment with primaquine, a substantial proportion of individuals $(0-59 \%$ after 4-6 months) undergo relapse and can act as an ongoing reservoir for transmission [21]. The relapse rate may in part be due to non-compliance with therapy, and some studies have demonstrated improved outcomes with directly observed therapy [21, 22]. Shorter courses have also been assessed which may improve adherence as the perceived benefit of treatment falls off as symptoms subside [22]. Recent studies have suggested that 7-day courses of primaquine may be as effective as a 14-days regimen [23, 24]. Tafenoquine, a novel 8-aminoquinoline administered as a single dose, has demonstrated to be non-inferior to primaquine in clinical trials [25] and as of this writing has been submitted to the U.S. Food and Drug Administration for registration. While confirmation of radical cure is desirable, no methods in clinical use exist currently to confirm eradication of latent infection [26].

Studies of $P$. vivax relapse in endemic areas are often difficult to design and interpret because subsequent P. vivax infections of study subjects may result either from reinfection or relapse, which are difficult to 
differentiate on a molecular basis. While highly similar or identical genotypes found in sequential infections (for example as determined by microsatellites) indicate likely relapse, finding a distinct genotype does not rule out relapse because of the possibility of relapse from a different hypnozoite clone acquired at a different time by an individual [27]. One such study came from Thailand where reinfection was considered unlikely due to low prevalence or impossible due to absence of transmission. Plasmodium vivax-infected subjects had been transported from malaria-endemic refugee camps along the Thai border to the non-endemic region of Bangkok. The authors found that the majority of relapses were characterised by genotypic strains distinct from the prior infection [28]. This observation was felt most likely to be due to inoculation of multiple different genotypes at the original infective event, with only a few parasites becoming detectable in the blood. Alternatively, different genotypes arose from hypnozoites reactivated over time that became detectable during follow up. In either case, these authors demonstrated the inherent difficulty of establishing the cause of recurrent infection in areas with endemic transmission and consequently the difficulty of identifying the contribution of relapse to ongoing transmission.

In addition to relapse of liver-stage infection, evidence has accumulated that low level blood stage infection may contribute to ongoing transmission. Infections detectable via molecular techniques but not by light microscopy are defined as sub-patent. Studies from the Peruvian Amazon have demonstrated that as many as $14 \%$ of individuals in a cross-sectional study were PCR positive for $P$. vivax with only a quarter of these exhibiting symptoms [8]. This compares to $2.9 \%$ of individuals having parasite detected by conventional microscopy [8]. These findings are supported by other studies performed in South America suggesting that PCR detection of $P$. vivax is approximately 5-7 times more sensitive than microscopy with most individuals not reporting symptoms $[6$, $8,29]$. But the question arises as to whether these subpatent infections are contributing to ongoing transmission. Alves et al. took fifteen asymptomatic individuals who were PCR positive only for $P$. vivax infection and undertook direct feeding and membrane feeding experiments with Anopheles darlingi with high parasitaemia symptomatic individuals as control. In total, only $1.2 \%$ of mosquitoes were infected by asymptomatic individuals compared to $22 \%$ of mosquitoes for the symptomatic carriers [7]. The authors concluded that while infection rates were lower, the much larger pool of patients and the likely longer duration of infection could mean that asymptomatic individuals represent a significant contribution to ongoing transmission.
A later study in the Peruvian Amazon followed 51 individuals with $P$. vivax infection identified through active or passive case detection [18]. Monthly follow-up included light microscopy, PCR and anti-circumsporozoite protein (CSP) antibody detection for 1 year after directly observed primaquine treatment. Twenty-nine of the 51 patients had 84 recurrent infections, of which only $26 \%$ were detectable by microscopy and just $21 \%$ reported symptoms. Sixteen of 29 patients with recurrent infection had more than one recurrence. Interestingly, about half of the 29 patients had recurrent infections that lasted 2 or more consecutive months all of which were sub-patent and asymptomatic. In three, the sub-patent infection went on to become patent. Considering all 51 patients, the overall person-infected-months was 13 per 100 person months of follow-up which equates to an average of 1.6 months per patient over the 1 year follow-up. The results suggest that $P$. vivax results in a substantial number of individuals maintaining ongoing transmission.

Since Alves et al. published landmark papers on the high prevalence of subclinical, subpatent $P$. vivax parasitaemia in Rôndonia State, Brazil [6], and the longitudinal potential contribution of such individuals to malaria transmission [7], similar observations have been made elsewhere in Amazonia [30] including Acre, Brazil $[13,31]$, the Loreto Department of Peru $[8,11,12]$ and Colombia [32, 33].

\section{Asymptomatic Plasmodium vivax parasitaemic humans, the reservoir of endemic transmission, and mechanisms of premunition}

Premunition in malaria-defined as protection against high parasitaemia and illness in the absence of completely eliminating infection-has long been known to occur $[34,35]$ despite what has been formally characterized as incomplete and "defective" immunity [36]; the natural history of malaria may lead to sterile immunity as well [37]. The major mechanism by which $P$. falciparum parasitaemia is controlled is widely thought to depend on the acquisition of polyclonal, protective antibodies, based on the classic study by Cohen in which the administration of immune gamma globulin was shown to lower but not eliminate $P$. falciparum parasitaemia [38].

Asymptomatic, parasitaemic individuals in endemic regions do not typically come to medical attention, hence they potentially remain infectious to mosquitoes. Asymptomatic parasitaemic individuals may have either patent or subpatent parasitaemia, and parasitaemia likely fluctuates over time and space. Hence asymptomatic parasitaemics likely move malaria parasites within the regions where they live and work [39]. The immunological mechanisms by which asymptomatic parasitaemia arises remain to be discovered, but generally speaking must 
be related to suppression of innate immune responses in the face of acquired immunity. Little is known about such mechanisms in P. vivax [40], but may relate in part to acquired antibodies against specific $P$. vivax attachment and invasion ligands (reviewed in refs. [15, 40]). Innate responses produce fever and other systemic manifestations of malaria-examples include TLR9-mediated responses to parasite DNA adsorbed to haemozoin [41] and parasite antigen-containing immune complexes [42] which produce inflammatory mediators (such as interleukin-1 and tumour necrosis factor-1) via inflammasome activation [43].

\section{State of malaria transmission-blocking vaccines}

Transmission-blocking immunity was first demonstrated in the 1970s with an avian model based on vaccinating chickens with Plasmodium gallinaceum gametes [44, 45]. The discovery of Pfs25 [46], Pfs48/45 [47] and Pfs230 [48, 49] as major surface antigens of $P$. falciparum zygotes and ookinetes led to considerable efforts to develop recombinant subunit transmission blocking vaccines (TBVs) [50], including early clinical trials $[51,52]$. P. falciparum transmission-blocking vaccine development continues [53].

On the basis of identifying the $P$. vivax orthologs of Pfs25, Pfs48/45 and Pfs230-C, TBVs based on these antigens is feasible but development of such a $P$. vivax TBV lags that of a $P$. falciparum TBV. Additional potential antigen targets of $P$. vivax TBV development remains to be explored, although the ability to obtain sexual stages of $P$. vivax (zygotes and ookinetes) to discover novel potential TBV antigens is feasible [54].

A $P$. vivax TBV is particularly attractive both because of the propensity of relapse in hypnozoite carriers and because the prevalence of asymptomatic parasitaemia in endemic regions is high. An anti-P. vivax TBV would either have to have sustained levels of antibodies over the time of potential relapse or populations would have to be revaccinated.

\section{Human infection by Plasmodium vivax and modulation of infectivity to mosquitoes}

A key, unaddressed gap in our understanding of the natural history of $P$. vivax infection is the relationship of infection to transmissibility of parasites. The important observation that most $P$. vivax relapses are asymptomatic [18] is key to understanding how asymptomaticallyinfected individuals may be key reservoirs of malaria transmission over space and time.

Experimental induction of antibodies against Plasmodium gametes that reduce Plasmodium infectivity to mosquitoes has been known for decades, including those of $P$. vivax, $[45,55,56]$. Later work producing monoclonal antibodies to block transmission of $P$. vivax found that at lower concentrations, transmission of infection to mosquito may actually be enhanced [57]. The authors took monoclonal antibodies raised through inoculation of female gametes into mice and mixed the serum with the blood of patients with acute vivax infection. The authors showed that transmission was reduced to below $10 \%$ of control with a concentrated monoclonal antibody solution. With serial dilutions, however, the effect was reversed with enhancement of infectivity to approximately two-fourfold above control. The effect of serum taken from patients in convalescence demonstrated a similar effect. Among 40 patients, 3 were found to have transmission enhancement effects with one inducing a 13-fold greater than control transmission. Using convalescent serum, the authors also demonstrated a time-post-infection effect with some serum initially blocking transmission but later enhancing transmission as anti-gamete antibody titres declined. The authors postulate potential mechanisms could include enhancement of fertilization or protection from antiparasitic factors in the mosquito blood meal. Using membrane feeding assays and direct feeds to experimentally infect laboratory-bred Anopheles tessellatus mosquitoes, studies of $P$. vivax-infected individuals in Sri Lanka demonstrated both naturallyacquired transmission-blocking and transmissionenhancing effects at different time points in relation to acute infection [58]. While these authors found that transmission-enhancing effects were observed early in $P$. vivax infection, transmission-blocking effects occurred later after initial infection. Interpretation of the data from these studies is limited because they were based on single time point sampling and not on serial sampling, so that the natural history of transmissionmodifying antibodies in individual subjects could not be ascertained.

Preliminary observations in the Peruvian Amazon in which laboratory-reared An. darlingi mosquitoes were infected via membrane feeding assays in the presence of serially-obtained sera (over 6 weeks) from subjects treated for acute $P$. vivax, have shown twofold increase in oocyst counts and fourfold increase in proportion of mosquitoes infected. These data further demonstrate the natural history of transmission-enhancing effects after acute $P$. vivax infection (McClean and Vinetz, manuscript in preparation).

These findings are striking in highlighting the complexity of immune response and host-parasite interaction. It also, shows the potential for complex and counter-intuitive responses to any potential vaccine dependent on immunologic response and waxing of immunity over time. 


\section{Characteristics of a successful transmission-blocking vaccine against Plasmodium vivax}

The concept of a vaccine blocking human infection of anopheline mosquitoes is an attractive approach to malaria control, elimination and eradication [59], but has not been robustly supported in the malaria community, at least in part because such a vaccine as a sole vaccine strategy has been considered to be "altruistic" but not of direct benefit to vaccinated individuals [46, 60-62]. Development has also been hampered by several major challenges including producing conformation of subunit vaccine proteins and the production of clinical grade material; the absence of validated standards and assays for efficacy; and the absence of a rapid pipeline of proof-of-principle human trials for testing lead candidates [63]. Based on homology with P. falciparum transmission-blocking vaccine candidates, the most promising $P$. vivax targets include Pvs48/45, Pvs28 and Pvs25 [64, 65].

The population impact of an effective transmission blocking vaccine is uncertain given the lack to date of phase 3 clinical trials but modelling suggests that a fivefold reduction in transmission is potentially achievable if $90 \%$ coverage could be implemented [53]. Modelling the impact of the best performing anti-Pvs 25 vaccine at the time, the author predicted a 1.6-fold reduction in transmission in a $90 \%$ coverage population. Importantly, the model suggests that vaccination against multiple antigens would reduce the concentration of antibody required to achieve a given level of protection.

\section{Conclusion}

Plasmodium vivax endemicity is maintained through a number of host-parasite interactions including relapse of hypnozoite stage infection, asymptomatic infection, early gametocyte appearance in the acute phase and immune interaction leading to enhanced transmission to the Anopheles vector. Disruption of these factors will be key to developing a strategy to eradicate infection and reduce the associated morbidity and mortality.

The ultimate goal of any malaria vaccine is to protect populations from malaria transmission arising from ongoing transmission and from reintroductions from movement of parasites into areas where transmission has been interrupted $[66,67]$. The present discussion emphasizes that the pathway forward for a $P$. vivax transmission-blocking vaccine rests on several observations and concepts: that asymptomatic parasitaemia in highly prevalent in endemic regions; that latent hypnozoite infection is usually associated with asymptomatic relapse; that infectious gametocytes appear early in $P$. vivax infection; and that transmission-enhancing antibodies develop as an intrinsic aspect of $P$. vivax infection.

\section{Authors' contributions}

TCSM and JMV both contributed to first and final drafts of the manuscript. Both authors read and approved the final manuscript.

\section{Acknowledgements}

JMV thanks the organizers of the 6th International Conference on Plasmodium vivax Research (ICPVR), held in June 11-14 2017, Manaus, Brazil for the opportunity to present the talk upon which this paper is based.

\section{Competing interests \\ The authors declare that they have no conflicts of interest or competing interests.}

Availability of data and materials

Not applicable.

\section{Consent for publication}

Not applicable.

Ethics approval and consent to participate Not applicable.

\section{Funding}

TCSM was supported by United States Public Health Service training grant from the National Institute of Allergy and Infectious Diseases, T32AI007036. This work was also supported in part by United States Public Health Service grant from the National Institute of Allergy and Infectious Diseases, U19AI089681 (JMV). TCSM and JMV declare no competing interests. All primary data and materials on which the present manuscript is based are included in the manuscript and are fully available.

\section{Publisher's Note}

Springer Nature remains neutral with regard to jurisdictional claims in published maps and institutional affiliations.

Received: 17 January 2018 Accepted: 17 February 2018

Published online: 21 February 2018

References

1. WHO. World Malaria Report 2017. Geneva: World Health Organization; 2017

2. Howes RE, Battle KE, Mendis KN, Smith DL, Cibulskis RE, Baird JK, et al. Global epidemiology of Plasmodium vivax. Am J Trop Med Hyg. 2016;95:15-34

3. Roberts DR, Laughlin LL, Hsheih P, Legters LJ. DDT, global strategies, and a malaria control crisis in South America. Emerg Infect Dis. 1997;3:295-302.

4. Griffing SM, Gamboa D, Udhayakumar V. The history of 20th century malaria control in Peru. Malar J. 2013:12:303.

5. Rosas-Aguirre A, Gamboa D, Manrique P, Conn JE, Moreno M, Lescano AG, et al. Epidemiology of Plasmodium vivax malaria in Peru. Am J Trop Med Hyg. 2016;95:133-44.

6. Alves FP, Durlacher RR, Menezes MJ, Krieger H, Silva LH, Camargo EP. High prevalence of asymptomatic Plasmodium vivax and Plasmodium falciparum infections in native Amazonian populations. Am J Trop Med Hyg. 2002;66:641-8

7. Alves FP, Gil LH, Marrelli MT, Ribolla PE, Camargo EP, Da Silva LH. Asymptomatic carriers of Plasmodium spp. as infection source for malaria vector mosquitoes in the Brazilian Amazon. J Med Entomol. 2005;42:777-9.

8. Roshanravan B, Kari E, Gilman RH, Cabrera L, Lee E, Metcalfe J, et al. Endemic malaria in the Peruvian Amazon region of lquitos. Am J Trop Med Hyg. 2003;69:45-52. 
9. Gething PW, Elyazar IR, Moyes CL, Smith DL, Battle KE, Guerra CA, et al. A long neglected world malaria map: Plasmodium vivax endemicity in 2010. PLoS Negl Trop Dis. 2012;6:e1814.

10. Price RN, von Seidlein L, Valecha N, Nosten F, Baird JK, White NJ. Global extent of chloroquine-resistant Plasmodium vivax: a systematic review and meta-analysis. Lancet Infect Dis. 2014;14:982-91.

11. Carrasco-Escobar G, Miranda-Alban J, Fernandez-Minope C, Brouwer KC, Torres K, Calderon M, et al. High prevalence of very-low Plasmodium falciparum and Plasmodium vivax parasitaemia carriers in the Peruvian Amazon: insights into local and occupational mobility-related transmission. Malar J. 2017;16:415.

12. Carrasco-Escobar G, Gamboa D, Castro MC, Bangdiwala SI, Rodriguez H, Contreras-Mancilla J, et al. Micro-epidemiology and spatial heterogeneity of P. vivax parasitaemia in riverine communities of the Peruvian Amazon: a multilevel analysis. Sci Rep. 2017;7:8082.

13. Barbosa S, Gozze AB, Lima NF, Batista CL, Bastos Mda S, Nicolete VC, et al. Epidemiology of disappearing Plasmodium vivax malaria: a case study in rural Amazonia. PLoS Negl Trop Dis. 2014;8:e3109.

14. Asih PB, Syafruddin D, Leake J, Sorontou Y, Sadikin M, Sauerwein RW, et al. Phenotyping clinical resistance to chloroquine in Plasmodium vivax in northeastern Papua, Indonesia. Int J Parasitol Drugs Drug Resist. 2011;1:28-32.

15. Mueller I, Galinski MR, Baird JK, Carlton JM, Kochar DK, Alonso PL, et al. Key gaps in the knowledge of Plasmodium vivax, a neglected human malaria parasite. Lancet Infect Dis. 2009;9:555-66.

16. Baird JK, Valecha N, Duparc S, White NJ, Price RN. Diagnosis and treatment of Plasmodium vivax malaria. Am J Trop Med Hyg. 2016;95:35-51.

17. Van den Eede P, Erhart A, Van der Auwera G, Van Overmeir C, Thang ND, le Hung $X$, et al. High complexity of Plasmodium vivax infections in symptomatic patients from a rural community in central Vietnam detected by microsatellite genotyping. Am J Trop Med Hyg. 2010;82:223-7.

18. Van den Eede P, Soto-Calle VE, Delgado C, Gamboa D, Grande T, Rodriguez $\mathrm{H}$, et al. Plasmodium vivax sub-patent infections after radical treatment are common in Peruvian patients: results of a 1-year prospective cohort study. PLoS ONE. 2011;6:e16257.

19. John GK, Douglas NM, von Seidlein L, Nosten F, Baird JK, White NJ, et al. Primaquine radical cure of Plasmodium vivax: a critical review of the literature. Malar J. 2012;11:280.

20. Grietens KP, Soto V, Erhart A, Ribera JM, Toomer E, Tenorio A, et al. Adherence to 7-day primaquine treatment for the radical cure of $P$. vivax in the Peruvian Amazon. Am J Trop Med Hyg. 2010;82:1017-23.

21. Durand S, Cabezas C, Lescano AG, Galvez M, Gutierrez S, Arrospide N, et al. Efficacy of three different regimens of primaquine for the prevention of relapses of Plasmodium vivax malaria in the Amazon Basin of Peru. Am J Trop Med Hyg. 2014;91:18-26.

22. Takeuchi R, Lawpoolsri S, Imwong M, Kobayashi J, Kaewkungwal J, Pukrittayakamee $\mathrm{S}$, et al. Directly-observed therapy (DOT) for the radical 14-day primaquine treatment of Plasmodium vivax malaria on the Thai-Myanmar border. Malar J. 2010;9:308.

23. Llanos-Cuentas A, Lacerda MV, Rueangweerayut R, Krudsood S, Gupta SK, Kochar SK, et al. Tafenoquine plus chloroquine for the treatment and relapse prevention of Plasmodium vivax malaria (DETECTIVE): a multicentre, double-blind, randomised, phase $2 \mathrm{~b}$ dose-selection study. Lancet. 2014;383:1049-58.

24. Cubi R, Vembar SS, Biton A, Franetich JF, Bordessoulles M, Sossau D, et al. Laser capture microdissection enables transcriptomic analysis of dividing and quiescent liver stages of Plasmodium relapsing species. Cell Microbiol. 2017;19(doi10):1111.

25. Chuquiyauri R, Molina DM, Moss EL, Wang R, Gardner MJ, Brouwer KC, et al. Genome-scale protein microarray comparison of human antibody responses in Plasmodium vivax relapse and reinfection. Am J Trop Med Hyg. 2015;93:801-9.

26. Imwong M, Snounou G, Pukrittayakamee S, Tanomsing N, Kim JR, Nandy A, et al. Relapses of Plasmodium vivax infection usually result from activation of heterologous hypnozoites. J Infect Dis. 2007;195:927-33.

27. da Silva-Nunes M, Moreno M, Conn JE, Gamboa D, Abeles S, Vinetz JM et al. Amazonian malaria: asymptomatic human reservoirs, diagnostic challenges, environmentally driven changes in mosquito vector populations, and the mandate for sustainable control strategies. Acta Trop. 2012;121:281-91.
28. Recht J, Siqueira AM, Monteiro WM, Herrera SM, Herrera S, Lacerda MVG. Malaria in Brazil, Colombia, Peru and Venezuela: current challenges in malaria control and elimination. Malar J. 2017;16:273.

29. Fontoura PS, Finco BF, Lima NF, de Carvalho JF, Vinetz JM Jr., Castro MC, et al. Reactive case detection for Plasmodium vivax malaria elimination in rural Amazonia. PLoS Negl Trop Dis. 2016;1 0:e0005221.

30. Saenz FE, Arevalo-Cortes A, Valenzuela G, Vallejo AF, Castellanos A, Poveda-Loayza AC, et al. Malaria epidemiology in low-endemicity areas of the northern coast of Ecuador: high prevalence of asymptomatic infections. Malar J. 2017;16:300.

31. Vallejo AF, Chaparro PE, Benavides Y, Alvarez A, Quintero JP, Padilla J, et al. High prevalence of sub-microscopic infections in Colombia. Malar J. 2015:14:201.

32. Perignon $J$, Druilhe P. Immune mechanisms underlying the premunition against Plasmodium falciparum malaria. Mem Inst Oswaldo Cruz. 1994;89(Suppl 2):51-3.

33. Druilhe P, Perignon JL. A hypothesis about the chronicity of malaria infection. Parasitol Today. 1997;13:353-7.

34. Struik SS, Riley EM. Does malaria suffer from lack of memory? Immunol Rev. 2004:201:268-90.

35. Soe S, Khin Saw A, Htay A, Nay W, Tin A, Than S, et al. Premunition against Plasmodium falciparum in a malaria hyperendemic village in Myanmar. Trans R Soc Trop Med Hyg. 2001;95:81-4.

36. Cohen S, McGregor IA, Carrington S. Gamma-globulin and acquired immunity to human malaria. Nature. 1961;192:733-7.

37. Parker BS, Paredes Olortegui M, Penataro Yori P, Escobedo K, Florin D, et al. Hyperendemic malaria transmission in areas of occupation-related travel in the Peruvian Amazon. Malar J. 2013;12:178.

38. Mueller I, Galinski MR, Tsuboi T, Arevalo-Herrera M, Collins WE, King CL. Natural acquisition of immunity to Plasmodium vivax: epidemiological observations and potential targets. Adv Parasitol. 2013;81:77-131.

39. Parroche P, Lauw FN, Goutagny N, Latz E, Monks BG, Visintin A, et al. Malaria hemozoin is immunologically inert but radically enhances innate responses by presenting malaria DNA to toll-like receptor 9. Proc Natl Acad Sci USA. 2007:104:1919-24.

40. Hirako IC, Gallego-Marin C, Ataide MA, Andrade WA, Gravina H, Rocha $B C$, et al. DNA-containing immunocomplexes promote inflammasome assembly and release of pyrogenic cytokines by CD14+ CD16+ CD64 high CD32 low inflammatory monocytes from malaria patients. MBio. 2015;6:e01605-15.

41. Kalantari P, DeOliveira RB, Chan J, Corbett Y, Rathinam V, Stutz A, et al. Dual engagement of the NLRP3 and AIM2 inflammasomes by plasmodiumderived hemozoin and DNA during malaria. Cell Rep. 2014;6:196-210.

42. Carter R, Chen D. Malaria transmission blocked by immunisation with gametes of the malaria parasite. Nature. 1976;263:57-60.

43. Gwadz RW. Malaria: successful immunization against the sexual stages of Plasmodium gallinaceum. Science. 1976;193:1150-1.

44. Kaslow DC, Quakyi IA, Syin C, Raum MG, Keister DB, Coligan JE, et al. A vaccine candidate from the sexual stage of human malaria that contains EGF-like domains. Nature. 1988;333:74-6.

45. Rener J, Graves PM, Carter R, Williams JL, Burkot TR. Target antigens of transmission-blocking immunity on gametes of Plasmodium falciparum. J Exp Med. 1983;158:976-81.

46. Quakyi IA, Carter R, Rener J, Kumar N, Good MF, Miller LH. The 230-kDa gamete surface protein of Plasmodium falciparum is also a target for transmission-blocking antibodies. J Immunol. 1987;139:4213-7.

47. Williamson KC. Pfs230: from malaria transmission-blocking vaccine candidate toward function. Parasite Immunol. 2003;25:351-9.

48. Kaslow DC. Transmission-blocking vaccines. Chem Immunol. 2002:80:287-307.

49. Da DF, Dixit S, Sattabonkot J, Mu J, Abate L, Ramineni B, et al. Anti-Pfs 25 human plasma reduces transmission of Plasmodium falciparum isolates that have diverse genetic backgrounds. Infect Immun. 2013;81:1984-9.

50. Wu Y, Ellis RD, Shaffer D, Fontes E, Malkin EM, Mahanty S, et al. Phase 1 trial of malaria transmission blocking vaccine candidates Pfs 25 and Pvs 25 formulated with montanide ISA 51. PLoS ONE. 2008;3:e2636.

51. Saul A. Mosquito stage, transmission blocking vaccines for malaria. Curr Opin Infect Dis. 2007;20:476-81.

52. Bounkeua V, Li F, Chuquiyauri R, Abeles SR, McClean CM, Neyra V, et al. Lack of molecular correlates of Plasmodium vivax ookinete development. Am J Trop Med Hyg. 2011;85:207-13. 
53. Carter R, Chen DH. Malaria transmission blocked by immunisation with gametes of the malaria parasite. Nature. 1976;263:57-60.

54. Munesinghe YD, Mendis KN, Carter R. Anti-gamete antibodies block transmission of human vivax malaria to mosquitoes. Parasite Immunol. 1986;8:231-8.

55. Peiris JS, Premawansa S, Ranawaka MB, Udagama PV, Munasinghe YD, Nanayakkara MV, et al. Monoclonal and polyclonal antibodies both block and enhance transmission of human Plasmodium vivax malaria. Am J Trop Med Hyg. 1988;39:26-32.

56. Gamage-Mendis AC, Rajakaruna J, Carter R, Mendis KN. Transmission blocking immunity to human Plasmodium vivax malaria in an endemic population in Kataragama, Sri Lanka. Parasite Immunol. 1992;14:385-96.

57. malERA consultative group on vaccines. A research agenda for malaria eradication: vaccines. PLoS Med. 2011;8:e1000398.

58. Moorthy V, Hill AV. Malaria vaccines. Br Med Bull. 2002;62:59-72.

59. Smith NJ. Malaria: the king is threatened. IDRC Rep. 1985;13:4-6.

60. Dinglasan RR, Jacobs-Lorena M. Flipping the paradigm on malaria transmission-blocking vaccines. Trends Parasitol. 2008;24:364-70.

61. Sauerwein RW, Richie TL. Malaria vaccines getting close to clinical reality. Vaccine. 2015:33:7423-4.

62. Tachibana M, Suwanabun N, Kaneko O, Iriko H, Otsuki H, Sattabongkot J, et al. Plasmodium vivax gametocyte proteins, Pvs48/45 and Pvs47, induce transmission-reducing antibodies by DNA immunization. Vaccine. 2015;33:1901-8.

63. Tsuboi T, Tachibana M, Kaneko O, Torii M. Transmission-blocking vaccine of vivax malaria. Parasitol Int. 2003;52:1-11.

64. O'Sullivan M, Kenilorea G, Yamaguchi Y, Bobogare A, Losi L, Atkinson JA, et al. Malaria elimination in Isabel Province, Solomon Islands: establishing a surveillance-response system to prevent introduction and reintroduction of malaria. Malar J. 2011;10:235.

65. Wesolowski A, Eagle N, Tatem AJ, Smith DL, Noor AM, Snow RW, et al. Quantifying the impact of human mobility on malaria. Science. 2012;338:267-70

66. O'Sullivan M, Kenilorea G, Yamaguchi Y, Bobogare A, Losi L, Atkinson JA, Vallely A, Whittaker M, Tanner M, Wijesinghe R. Malaria elimination in Isabel Province, Solomon Islands: establishing a surveillance-response system to prevent introduction and reintroduction of malaria. Malar J. 2011;10:235.

67. Wesolowski A, Eagle N, Tatem AJ, Smith DL, Noor AM, Snow RW, Buckee CO. Quantifying the impact of human mobility on malaria. Science. 2012;338:267-70

\section{Submit your next manuscript to BioMed Central and we will help you at every step:}

- We accept pre-submission inquiries

- Our selector tool helps you to find the most relevant journal

- We provide round the clock customer support

- Convenient online submission

- Thorough peer review

- Inclusion in PubMed and all major indexing services

- Maximum visibility for your research

Submit your manuscript at www.biomedcentral com/submit 